\title{
NPR-A regulates self-renewal and pluripotency of embryonic stem cells
}

\author{
EM Abdelalim ${ }^{*, 1,2}$ and I Tooyama ${ }^{1}$
}

Self-renewal and pluripotency of embryonic stem (ES) cells are maintained by several signaling cascades and by expression of intrinsic factors, such as Oct4, Nanog and Sox2. The mechanism regulating these signaling cascades in ES cells is of great interest. Recently, we have demonstrated that natriuretic peptide receptor A (NPR-A), a specific receptor for atrial and brain natriuretic peptides (ANP and BNP, respectively), is expressed in pre-implantation embryos and in ES cells. Here, we examined whether NPR-A is involved in the maintenance of ES cell pluripotency. RNA interference-mediated knockdown of NPR-A resulted in phenotypic changes, indicative of differentiation, downregulation of pluripotency factors (such as Oct4, Nanog and Sox2) and upregulation of differentiation genes. NPR-A knockdown also resulted in a marked downregulation of phosphorylated Akt. Furthermore, NPR-A knockdown induced accumulation of ES cells in the G1 phase of the cell cycle. Interestingly, we found that ANP was expressed in self-renewing ES cells, whereas its level was reduced after ES cell differentiation. Treatment of ES cells with ANP upregulated the expression of Oct4, Nanog and phosphorylated Akt, and this upregulation depended on NPR-A signaling, because it was completely reversed by pretreatment with either an NPR-A antagonist or a cGMP-dependent protein kinase inhibitor. These findings provide a novel role for NPR-A in the maintenance of self-renewal and pluripotency of ES cells. Cell Death and Disease (2011) 2, e127; doi:10.1038/cddis.2011.10; published online 10 March 2011

Subject Category: Experimental medicine

Embryonic stem (ES) cells are pluripotent and self-renewing cells generated by cellular outgrowth of pre-implantation embryos. ${ }^{1,2}$ They have the ability to differentiate into the representative cell types of all three germ layers of the embryo. ${ }^{3}$ These unique properties make them exceptionally valuable for cell replacement therapies and for regenerative medicine, and control of self-renewal and pluripotency of ES cells is important for their potential.

The pluripotent identity of ES cells is controlled by a group of transcription factors. ${ }^{4-8}$ Within this group, the transcription factors Oct4, Nanog and Sox2 contribute to the hallmark characteristics of ES cells by activation of target genes that encode pluripotency and self-renewal mechanisms, as well as by repression of signaling pathways that promote differentiation. ${ }^{8}$ In addition, ES cell pluripotency requires inputs from extrinsic factors and their downstream effectors. ${ }^{9}$ However, the molecular mechanisms that control pluripotency and differentiation of ES cells are largely unknown. Therefore, it is important to elucidate the transcriptional networks surrounding Oct4, Nanog and Sox2.

Natriuretic peptide receptor A (NPR-A) is the receptor for atrial natriuretic peptide (ANP) and brain natriuretic peptide (BNP). ANP and BNP, members of the natriuretic peptide family, have been extensively studied for their functions in regulating blood pressure. ${ }^{10,11}$ In addition to this cardiovascular profile, there is evidence that suggests that ANP can also act as an autocrine/paracrine factor. ${ }^{12}$ Previous studies have reported that ANP has apoptotic, ${ }^{13}$ anti-apoptotic, antihypertrophic, ${ }^{14}$ anti-mitogenic ${ }^{15}$ and mitogenic ${ }^{16}$ effects, and has a role in tumor cell growth. ${ }^{17}$ Both ANP and BNP signal through NPR-A or natriuretic peptide receptor B (NPR-B) by increasing cGMP and by activating cGMP-dependent protein kinase (PKG), which in turn can activate different downstream effectors involved in cell growth, apoptosis, proliferation and inflammation. ${ }^{10,18}$ ANP does not bind to NPR-B, but some mitogenic effects of BNP are mediated through its binding to NPR-B. ${ }^{19}$

Previous studies with mice using genetic disruption of the NPR-A gene have been widely used to examine the role of natriuretic peptide signaling in cardiac functions. ${ }^{20-22}$ These studies focused on the effect of disrupted NPR-A in adult animals only. However, a critical requirement of NPR-A in early development has been recently documented. ${ }^{23}$ Recently, we showed that NPR-A is expressed at high levels in pre-implantation embryos and in undifferentiated murine ES cells, which is rapidly downregulated after their differentiation, ${ }^{24}$ suggesting involvement of NPR-A in regulating early development.

As no role has been established for NPR-A in ES cells, we aimed to selectively knock down NPR-A expression in murine ES cells using an RNA interference approach to evaluate its role in self-renewal and pluripotency of ES cells. Furthermore, we examined the effect of exogenous ANP on ES cell

\footnotetext{
${ }^{1}$ Molecular Neuroscience Research Center, Shiga University of Medical Science, Setatsukinowa-cho, Otsu, Shiga 520-2192, Japan and ${ }^{2}$ Department of Cytology \& Histology, Faculty of Veterinary Medicine, Suez Canal University, Ismailia, Egypt

${ }^{*}$ Corresponding author: EM Abdelalim, Molecular Neuroscience Research Center, Shiga University of Medical Science, Setatsukinowa-cho, Otsu 520-2192, Japan. Tel: + 8177548 2402; Fax: + 8177548 2402; E-mail: essam_abdelalim@yahoo.com or essam @belle.shiga-med.ac.jp Keywords: ANP; ES cells; natriuretic peptide receptor A; RNA interference; self-renewal

Abbreviations: ANP, atrial natriuretic peptide; AP, alkaline phosphatase; BNP, brain natriuretic peptide; EBs, embryoid bodies; ES, embryonic stem; LIF, leukaemia inhibitory factor; NPR-A, natriuretic peptide receptor A; NPR-B, natriuretic peptide receptor B; PI3K, phosphoinsitide 3-kinase; PKG, protein kinase G; RA, retinoic acid; siRNA, small interfering RNA

Received 20.12.10; revised 26.1.11; accepted 01.2.11; Edited by P Salomoni
} 
pluripotency factors. The data presented here show that knockdown of NPR-A in murine ES cells results in differentiation of ES cells in the presence of the leukemia-inhibitory factor (LIF). Conversely, exogenous ANP activated the expression of the pluripotency factors, Oct4 and Nanog, through the NPR-A pathway.

\section{Results}

Knockdown of NPR-A leads to loss of self-renewal and pluripotency in ES cells. Previously, we have shown that NPR-A is specifically expressed in undifferentiated ES cells. ${ }^{24}$ To confirm these data, we examined the expression of NPR-A under self-renewal and differentiation conditions. Double-immunofluorescence analysis showed that NPR-A was highly expressed in undifferentiated ES (Oct4-positive) cells, which were cultured in the presence of LIF, and that expression was downregulated upon differentiation induced by culturing ES cells without LIF for 5 days. Differentiated cells, which were negative for Oct4 expression, were also negative for NPR-A expression (Supplementary Figure 1), indicating that NPR-A is specifically expressed in selfrenewing ES cells.

To determine the role of NPR-A in murine ES cells, a smallinterfering RNA (siRNA)-based technique using two independent siRNAs targeting different regions of NPR-A mRNA was used to specifically knock down the NPR-A gene in ES cells maintained in a feeder-free culture. Before siRNA transfection, the undifferentiated status of murine ES cells used for transfection was confirmed by flow cytometry and immunofluorescence (for Oct4-positive cells) (data not shown). Knockdown efficiency was analyzed at $48 \mathrm{~h}$ after transfection of siRNA.

Both reverse transcription-PCR (RT-PCR) and real-time PCR revealed a marked reduction in the level of NPR-A mRNA at $48 \mathrm{~h}$ after transfection in ES cells, which were transfected with NPR-A-targeting siRNA (NPR-A siRNA), compared with ES cells that were transfected with a
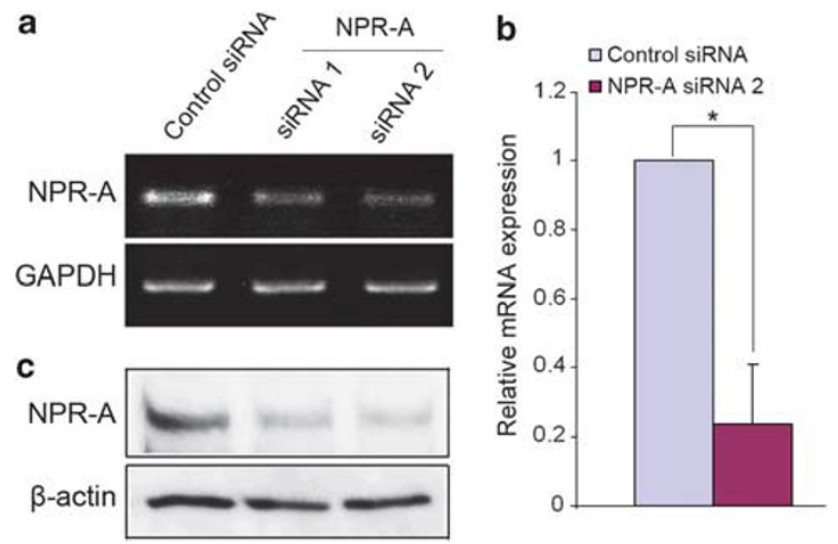

Figure 1 NPR-A small-interfering RNA (siRNA) induces efficient knockdown of NPR-A in murine ES cells. (a) RT-PCR analysis of ES cells transfected with control siRNA or NPR-A siRNAs, showing knockdown of the NPR-A gene $48 \mathrm{~h}$ after siRNA transfection. GAPDH was used as the internal control. (b) Real-time PCR analysis of ES cells treated with control siRNA or NPR-A siRNA2. (c) Western blot analysis of ES cells treated as described in panel a, showing a reduced level of the NPR-A protein $48 \mathrm{~h}$ after siRNA transfection. $\beta$-Actin was used as a loading control. Data represent mean \pm S.D. $(n=3) ;{ }^{*} P<0.05$ (two-tailed $t$-test) nontargeting siRNA (control siRNA) (Figures 1a and b). This finding was confirmed by a reduction in protein levels observed in western blots (Figure 1c).

We also evaluated colony morphology and alkaline phosphatase (AP) activity in ES cells cultured in the presence of LIF. Two days after transfection, NPR-A siRNA-treated cells exhibited a striking change in morphology with the presence of colonies composed of much larger, flattened cells typical of differentiating cells (Figure 2a). AP staining, which is indicative of the self-renewal property of ES cells, was markedly reduced in ES cells treated with NPR-A siRNA in comparison with those treated with control siRNA (Figure 2b).

Next, we examined the expression of the pluripotency factor Oct4 by immunostaining at $72 \mathrm{~h}$ after siRNA transfection. As shown in Figure 2c, Oct4 expression was decreased in ES cells treated with NPR-A siRNA than in control siRNA-treated cells. To provide a more quantitative analysis, we used immunoblotting to examine the expression of Oct4 and Nanog. We found that NPR-A siRNA-treated cells showed a marked reduction in the level of Oct4 and Nanog, which are the master regulators of ES cell self-renewal and pluripotency, compared with control siRNA-treated cells (Figure 2d). Both siRNAs targeting NPR-A caused a similar change in morphology, AP staining, Oct4 expression and Nanog expression (Figures 2a-d).

To examine the self-renewal of NPR-A knockdown ES cells at the single cell level, 2 days after siRNA transfection, ES cells were seeded at low density (1000 or 2000 cells per $35-\mathrm{mm}$ dish) in the presence of LIF for 6 days to form secondary ES cell colonies (Figure $2 \mathrm{e}$ ). The number of formed colonies of NPR-A siRNA-treated ES cells was significantly reduced than that of control siRNA-treated cells, and the majority of colonies formed by NPR-A siRNA-treated cells appeared differentiated when compared with control siRNAtreated cells. To confirm these results, AP staining was performed (Figure 2f). Quantification of AP-positive colonies showed that $>90 \%$ of control siRNA-treated cells were undifferentiated, whereas NPR-A siRNA-treated cells showed a significant decrease in the proportion of undifferentiated colonies ( $\sim 46 \%$ ) (Figure $2 \mathrm{f})$. These findings suggest that NPR-A is important for self-renewal of ES cells.

NPR-A knockdown downregulates pluripotency genes and upregulates differentiation genes in murine ES cells. To assess the differentiation state of NPR-A knockdown cells, we assayed for changes in the expression of key pluripotency genes (such as Oct4, Nanog and Sox2) that are considered a part of the core set of factors associated with the maintenance of pluripotency and self-renewal in ES cells. ${ }^{4-7}$ RT-PCR and real-time PCR analyses of all these genes showed a decreased level of expression in NPR-A-deficient cells when compared with control siRNA-treated cells (Figures $3 a$ and $b$ ). These results indicate that NPR-A has a role in regulating ES cell pluripotency genes.

We then examined changes in the expression levels of early differentiation marker genes such as GATA-4 and GATA-6 for the extraembryonic endoderm, $A F P$ for the visceral endoderm, Brachyury for mesoderm, nestin for ectoderm and Cdx2, Hand1 and Eomes for trophectoderm. All these genes 
a
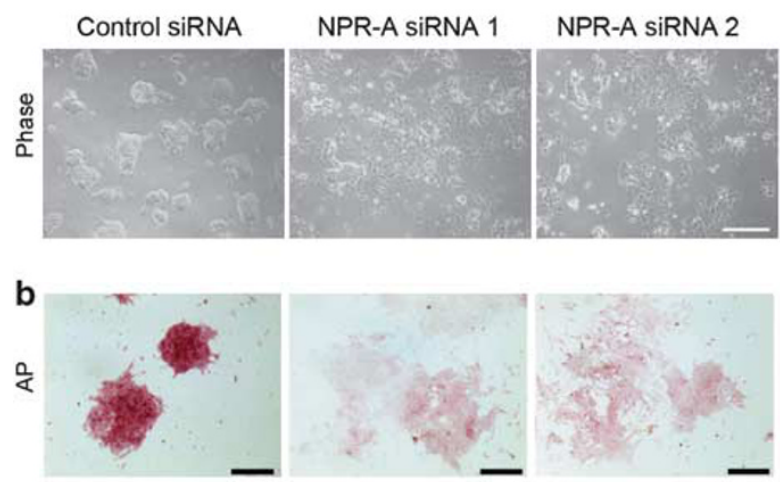

c Control siRNA

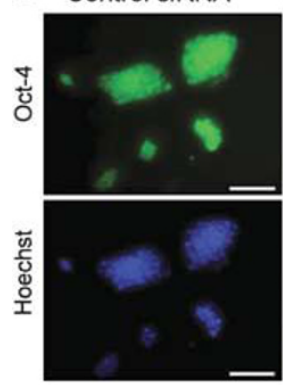

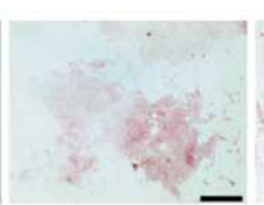

NPR-A SiRNA 1

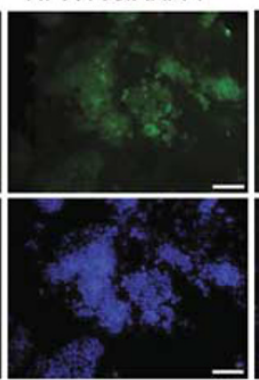

NPR-A siRNA 2

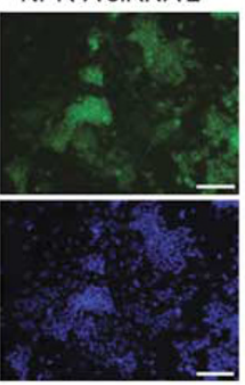

d

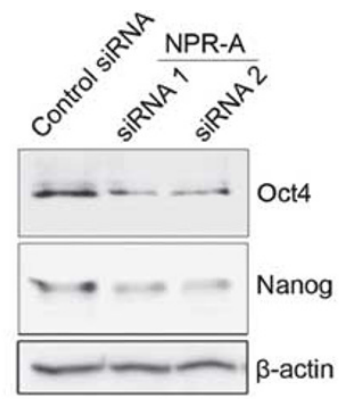

e

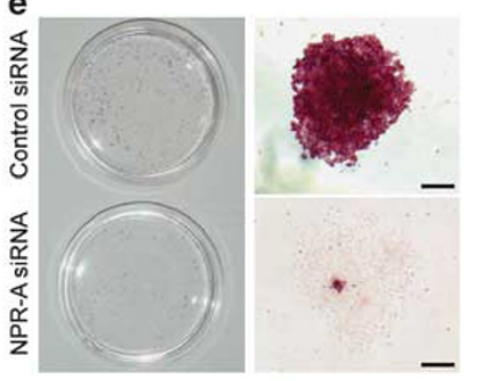

f

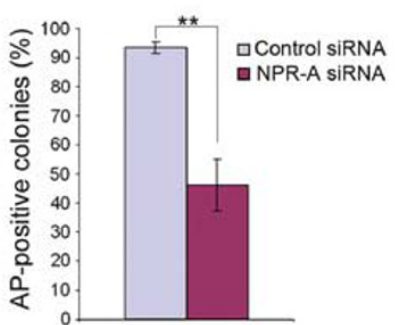

Figure 2 Knockdown of NPR-A reduces the potential for ES cell self-renewal. (a) Morphology of ES cells treated with control siRNA or NPR-A siRNAs at 2 days after transfection in the presence of LIF. (b) Self-renewal assay, AP-staining of ES cells treated as described in panel a, 4 days after transfection. (c) Immunofluorescence images of ES cells treated as in panel a for $72 \mathrm{~h}$, stained with antibodies against Oct4 and counterstained with Hoechst reagent. (d) Western blot analysis of ES cells treated as in panel a, showing reduced protein levels of Oct4 and Nanog $72 \mathrm{~h}$ after siRNA transfection. $\beta$-Actin was used as a loading control. (e) Left panels, photographs of the formed colonies after 6 days from culturing at low density in the presence of LIF, stained with AP. AP staining of cells treated as in panel a. It must be noted that the staining intensity of NPR-A siRNA-treated cells is decreased. Right panels, high-magnification images of AP staining. (f) Graph showing quantification of the percentages of AP-positive colonies treated as described in panel e. Data represent mean \pm S.D. $(n=2)$ and significant values are indicated; ${ }^{* \star} P<0.01$, compared with control (two-tailed $t$-test). Scale bar $=50 \mu \mathrm{m}$

were upregulated in ES cells treated with NPR-A siRNA compared with control siRNA (Figures $3 c$ and $d$ ). This increase in multi-lineage-specific genes suggests that cells were differentiated along several different pathways.

We next examined the differentiation potential of NPR-Adeficient ES cells by embryoid body (EB) formation in the presence of retinoic acid (RA) (Supplementary Figure 2). NPR-A siRNA-derived EBs were much smaller in size compared with EBs derived from control siRNA-treated cells (Supplementary Figure $2 b$ ). Although EBs derived from both control siRNA and NPR-A siRNA started expressing multiple differentiation markers as examined by RT-PCR, EBs derived from NPR-A-deficient cells expressed greater levels of endoderm (GATA-4, GATA-6 and AFP), ectoderm (nestin) and trophectoderm (Hand1 and Eomes) markers (Supplementary Figure 2d). However, Brachyury, a marker of mesoderm, was slightly reduced by NPR-A knockdown, indicating that NPR-A knockdown predisposes ES cells to endodermal, ectodermal and trophectodermal differentiation. Pluripotency markers (such as Oct4 and Nanog) were detected at higher levels in NPR-A siRNA-derived EBs than in those of control siRNA-derived EBs (Supplementary
Figures 2c and e), most likely as a result of the incomplete differentiation of a subset of cells.

NPR-A knockdown results in suppression of G1-S transition. As a consequence of impairment in selfrenewing properties, we examined whether NPR-A knockdown could also affect the proliferation of ES cells. ES cells treated with control siRNA or NPR-A siRNA were counted after $48 \mathrm{~h}$ from siRNA transfection. NPR-A siRNAtreated cells showed a significant reduction in cell number than did control siRNA-treated cells (Figure 4a), consistent with the fact that they differentiate spontaneously.

Next, we evaluated whether downregulation of NPR-A affected the ES cell cycle. We analyzed the phases of the cell cycle $48 \mathrm{~h}$ after siRNA transfection. The knockdown of NPR-A expression induced a substantial accumulation of ES cells in the G0/G1 phase, when compared with control siRNA-treated cells (Figures $4 b$ and c; Supplementary Figure 3). In addition, NPR-A knockdown reduced the proportion of cells in the $S$ phase (Figures $4 b$ and $c$ ). Thus, NPR-A knockdown in ES cells inhibits ES cell proliferation by blocking cell-cycle progression in G0/G1 and by preventing G1-S transition. 


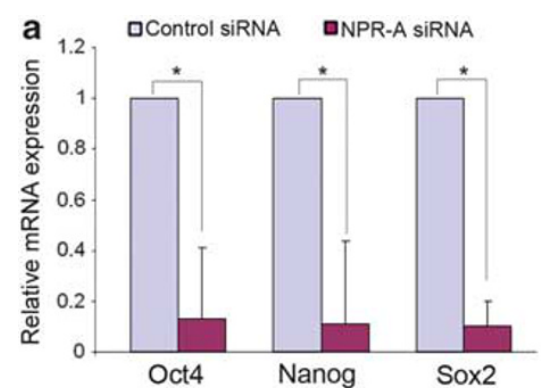

C

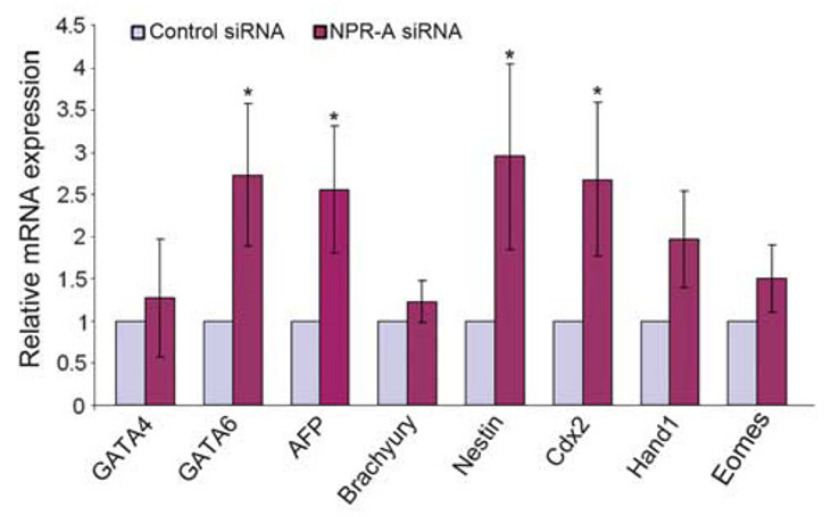

b

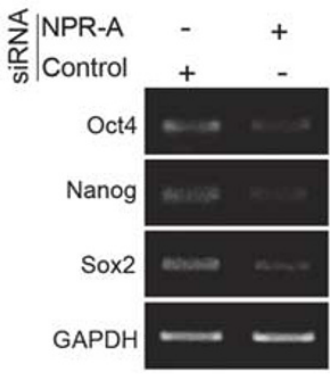

d

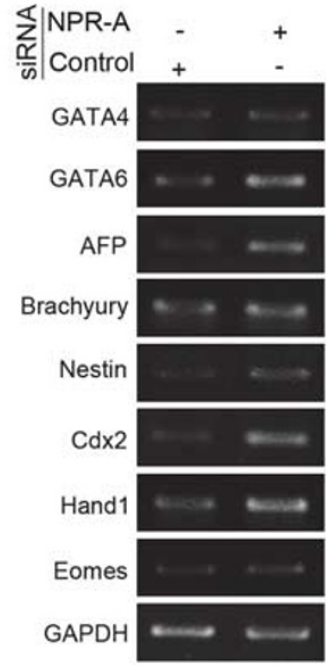

Figure 3 NPR-A knockdown decreases ES pluripotency genes and upregulates differentiation genes. (a) Real-time PCR of mRNA levels of pluripotency genes (Oct4, Nanog and Sox2) after treatment of ES cells with control siRNA or NPR-A siRNA. (b) RT-PCR analysis of ES cells treated as described in panel a, showing expression of stem cell marker genes. GAPDH was used as the internal control. (c) Real-time PCR of ES cells treated as described in panel a, showing mRNA levels of the early differentiation genes, GATA-4 (GATA-binding protein 4), GATA-6 (GATA-binding protein 6), AFP ( $\alpha$-fetoprotein), Brachyury, nestin, Cdx2 (caudal-type homeobox 2), Hand1 (heart and neural crest derivatives expressed transcript 1) and Eomes (eomesodermin homolog). (d) RT-PCR analysis of ES cells treated as described in panel a, showing mRNA levels of the early differentiation genes. GAPDH was used as the internal control. Data represent mean \pm S.D. $(n=3)$; ${ }^{*} P<0.05$ (two-tailed $t$-test)

To further understand how loss of NPR-A could regulate G1-S transition, we performed RT-PCR and real-time PCR analyses of cells treated with control siRNA or NPR-A siRNA for genes involved in the G1-S transition. Consistent with changes in the cell cycle, knockdown of NPR-A led to a downregulation in the transcription of cyclin D1, one of the G1-phase cyclins, whereas transcription and proteins of p21 were upregulated (Figures $4 \mathrm{~d}-\mathrm{f}$ ). These results suggest that NPR-A suppresses inhibitors of the G1-S transition, allowing rapid transition from mitosis to the $S$ phase.

NPR-A knockdown inhibits PI3 signaling. We investigated the effect of NPR-A knockdown on the phosphoinositide 3-kinase (PI3K) signaling pathway, which is critical for maintenance of murine ES cell pluripotency and selfrenewal, ${ }^{25,26}$ by analyzing phosphorylation levels of Akt. We found that knockdown of NPR-A dramatically reduced Akt phosphorylation in comparison with controls (Figures $4 \mathrm{~g}$ and $\mathrm{h}$ ).

ANP is expressed in ES cells and promotes ES cell pluripotency. We found that ANP was expressed in undifferentiated ES cells (Oct4-positive cells), which were cultured in the presence of LIF, as examined by immunofluorescence (Figure 5a), and that the expression was downregulated upon differentiation, induced by culturing
ES cells without LIF for 5 days. The differentiated cells, which were negative for Nanog expression, were also negative for ANP expression (Figure 5a). Similar results were obtained by western blotting (Figure $5 b$ ). These results indicate that ANP is specifically expressed in self-renewing ES cells. ANP knockdown in ES cells affected ES cell self-renewal (Supplementary Information, Supplementary Figure 4).

As NPR-A is expressed by undifferentiated ES cells, ${ }^{24}$ it suggests that these cells may be responsive to the NPR-A ligand, ANP. ES cells cultured in a serum-free medium were treated with $2.5 \mu \mathrm{M}$ ANP or vehicle, and then the expression of Oct4, Nanog and phosphorylated Akt was examined.

Real-time PCR analysis of ES cell pluripotency marker genes, including Oct4 and Nanog, demonstrated a significant upregulation in the mRNA levels of Oct4 and Nanog, $12 \mathrm{~h}$ after ANP treatment (Figure 5c). Furthermore, western blotting showed a marked increase in the levels of Oct4 and a smaller increase in Nanog levels in ANP-treated ES cells, compared with control-treated cells after 12 and $24 \mathrm{~h}$ (Figure 5d). In addition, western blot analysis showed a marked upregulation in the expression of phosphorylated Akt examined at $12 \mathrm{~h}$ from ANP treatment (Figures $5 e$ and $\mathrm{f}$ ).

To determine the relationship between ANP/NPR-A and the PI3K signaling pathway, we used LY294002, a specific 

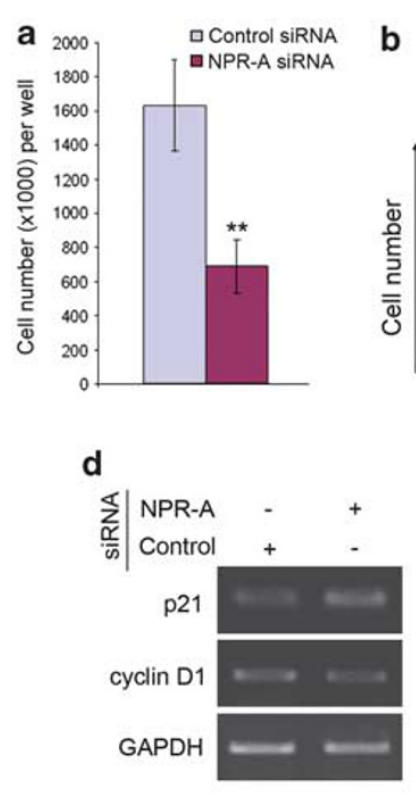

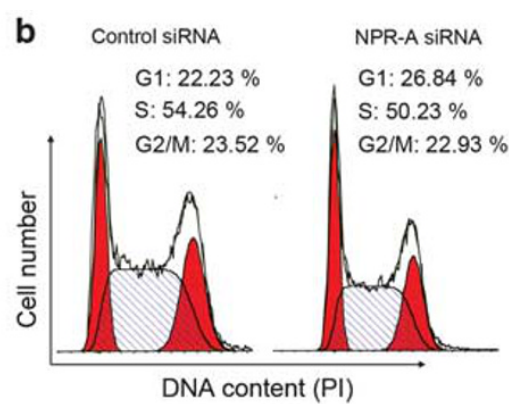

DNA content (PI)

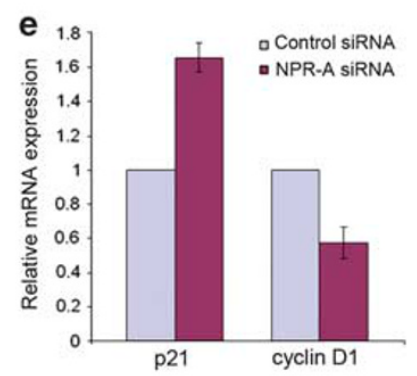

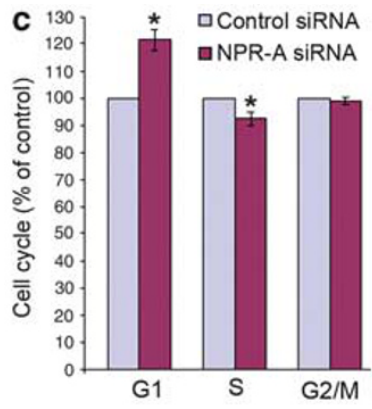

f

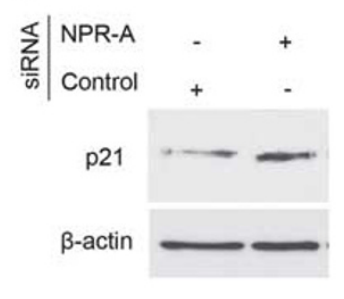

g

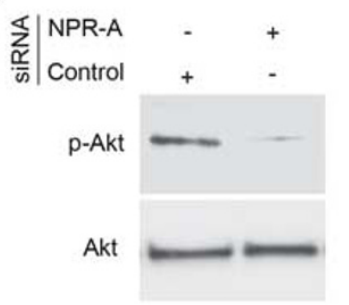

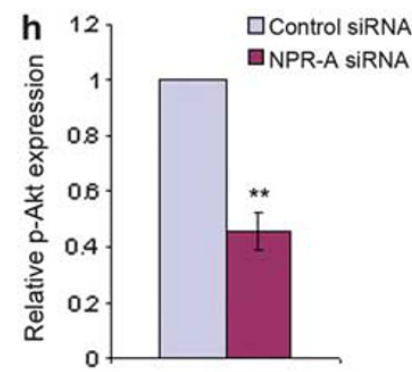

Figure 4 NPR-A knockdown reduced ES cell propagation, prolonged the G1 phase and upregulated phosphorylated Akt in murine ES cells. (a) Quantification of ES cells $48 \mathrm{~h}$ after transfection with control siRNA or NPR-A siRNA $(n=4)$. (b) Effect of NPR-A knockdown on cell-cycle progression, showing the percentages of cells in the G1, S and G2/M phases of the cell cycle. (c) Quantification of cell-cycle phases $(n=3)$. (d) RT-PCR analysis of p21 and cyclin D1 mRNA $48 \mathrm{~h}$ after ES cell transfection with control siRNA or NPR-A siRNA. (e) Real-time PCR analysis of ES cells treated as described in panel d. (f) Western blot analysis for p21 $48 \mathrm{~h}$ after siRNA transfection. (g) Western blot analysis for phosphorylated Akt (Ser473; $\mathrm{p}$-Akt) and total Akt $48 \mathrm{~h}$ after siRNA transfection. $\beta$-Actin was used as a loading control. (h) Relative levels of the p-Akt protein were quantified after normalization to total Akt. Data represent mean \pm S.D. $(n=3) ;{ }^{*} P<0.05$ or ${ }^{\star \star} P<0.01$ (two-tailed $t$-test)

inhibitor of the PI3K pathway, to inhibit PI3K signaling. ${ }^{26}$ ES cells cultured in the presence of LIF were treated for 3 days with ANP, LY294002 or ANP and LY294002 together. Control and ANP-treated cells maintained undifferentiated morphology as indicated by strong AP staining, whereas ES cells treated with LY294002 alone showed extensive differentiation with negative AP staining. ANP- and LY294002-treated cells showed a moderate differentiation and several colonies were AP positive (Figure $5 \mathrm{~g}$ ).

PI3K signaling is required for Nanog expression, as a PI3K inhibitor efficiently represses Nanog expression in ES cells. ${ }^{26}$ In agreement, Nanog expression markedly reduced in cells treated with LY294002 compared with control cells at $18 \mathrm{~h}$. However, ES cells treated with ANP and LY294002 maintained Nanog expression at a higher level than did those cultured in the presence of LY294002 alone (Figures 5h and i), indicating that the activation of NPR-A partially sustained ES cell pluripotency in the presence of the PI3K inhibitor by activating the Nanog expression.

Involvement of ANP receptor-mediated signaling in the upregulation of Oct 4 and Nanog was confirmed using anantin (a specific NPR-A antagonist), which blocked the effect of
ANP on the expression of Oct4 and Nanog when ES cells were treated with $300 \mathrm{nM}$ anantin before ANP exposure (Figures $6 a$ and $b$ ). Furthermore, to test the possibility of the involvement of PKG signaling in mediating ANP effect on the expression of Oct4 and Nanog, we used KT5823 (a specific inhibitor of PKG). Pretreatment with $2 \mu \mathrm{M}$ of the PKG inhibitor $30 \mathrm{~min}$ before ANP treatment, blocked the upregulation of Oct4 and Nanog (Figures $6 \mathrm{c}$ and d), suggesting the involvement of the NPR-A/PKG pathway in this process.

The effect of NPR-A/cGMP inhibitors on ES cell pluripotency was examined by culturing ES cells in the presence of anantin or KT5823 for 3 days in the serum-free medium. Both inhibitors caused downregulation in the protein levels of Oct4 and Nanog (Figures $6 e$ and f). These results confirm the involvement of the NPR-A/cGMP pathway in ES cell pluripotency (Supplementary Figure 5).

\section{Discussion}

Here, we present evidence demonstrating that NPR-A is critical for self-renewal and pluripotency of murine ES cells. Our novel findings are summarized as follows: (1) Reduction 

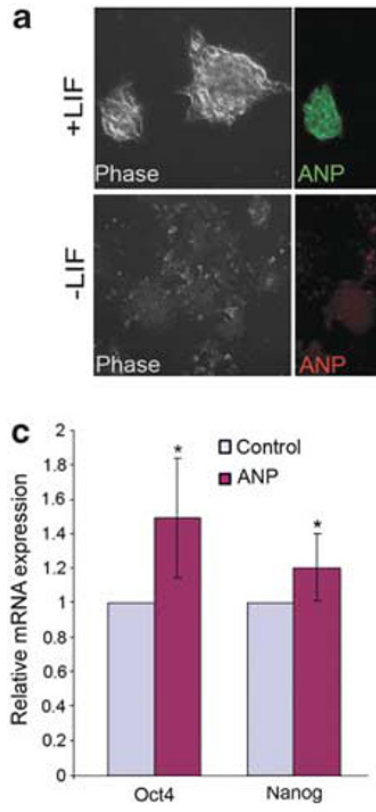
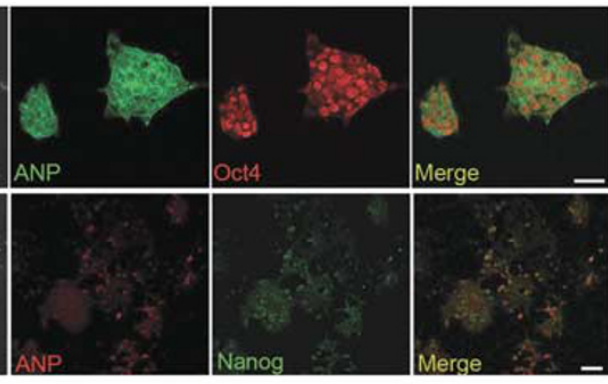

d

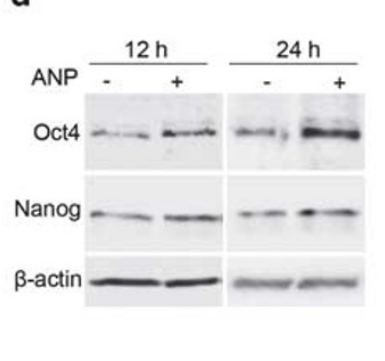

e b

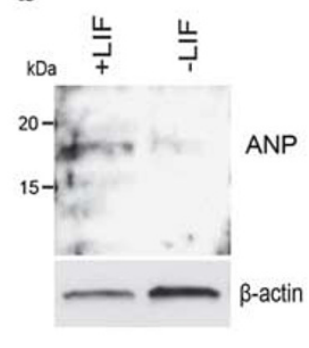

g

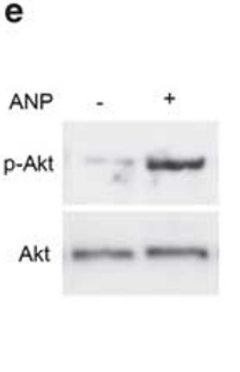

f
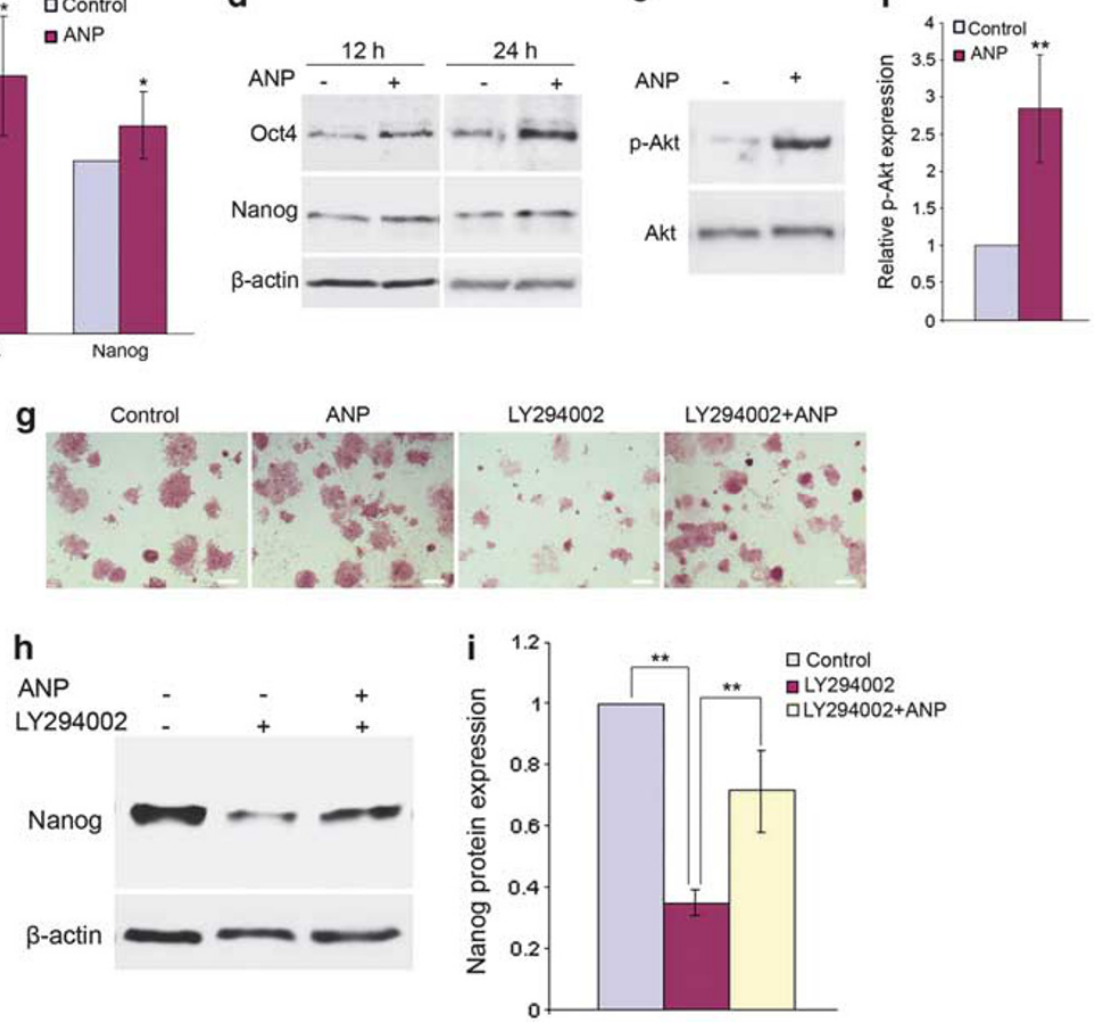

Figure 5 ANP is expressed in murine ES cells, and exogenous ANP stimulates ES cell pluripotency factors. (a) Upper panels, double-immunofluorescence images of ES cells cultured in the presence of LIF, stained with antibodies against the ES cell markers, Oct4 and ANP. Lower panels, double-immunofluorescence images of ES cells cultured in the absence of LIF for 5 days, stained with antibodies against Nanog and ANP. It must be noted that ANP signals are downregulated upon differentiation. (b) Western blot analysis for ANP of ES cells treated as described in panels $\mathbf{a}$ and $\mathbf{b}$. (c) Real-time PCR analysis of mRNA levels for pluripotency genes $12 \mathrm{~h}$ after treatment of ES cells with $2.5 \mu \mathrm{M}$ ANP. (d) Western blot analysis of Oct4 and Nanog in ES cells treated with ANP for 12 or $24 \mathrm{~h}$. $\beta$-Actin is shown as a control for loading. (e) Western blot analysis for phosphorylated Akt (Ser473; p-Akt) and total Akt $12 \mathrm{~h}$ after treatment of ES cells with ANP. (f) Relative levels of the $\mathrm{p}$-Akt protein were quantified after normalized to total Akt. (g) ES cells were treated with LY294002, ANP or ANP + LY294002 for 3 days, followed by staining for alkaline phosphatase (AP). (h) Western blot analysis of Nanog in ES cells treated with LY294002 or ANP + LY294002 for $18 \mathrm{~h}$. (i) Quantitative analysis of the western blots as shown in panel h. Data represent mean \pm S.D. ( $n=3$ ). ${ }^{*} P<0.05$ or ${ }^{*} P<0.01$ (two-tailed $t$-test)

of NPR-A expression by siRNA in murine ES cells leads to differentiation of ES cells in the presence of LIF. This differentiation is accompanied by reduced expression of Oct4, Nanog and Sox2, and induced expression of early differentiation markers. (2) Knockdown of NPR-A accumulates ES cells in the G1 phase of the cell cycle, and downregulates phosphorylated Akt. (3) ANP is expressed in undifferentiated ES cells, whereas its expression is reduced after ES cell differentiation. (4) Exogenous ANP stimulates ES cell pluripotency factors (namely Oct4 and Nanog) and upregulates phosphorylated Akt, involving the NPR-A/PKG pathway.
We have previously shown that NPR-A is expressed in pluripotent cells of the inner cell mass of the blastocyst and in murine ES cells, ${ }^{24}$ which implies that its loss may affect development in vivo. These results are in agreement with a recent study in mice in which ablation of the NPR-A gene led to reduced viability of the embryo. ${ }^{23}$ The linkage between NPR-A expression in ES cells and embryonic development strongly suggests that signaling through this receptor may have a role in maintaining ES cell pluripotency. Interestingly, our data revealed that ANP, the ligand for NPR-A, is specifically expressed in self-renewing ES cells, suggesting its involvement in mediating NPR-A function in ES cells. 

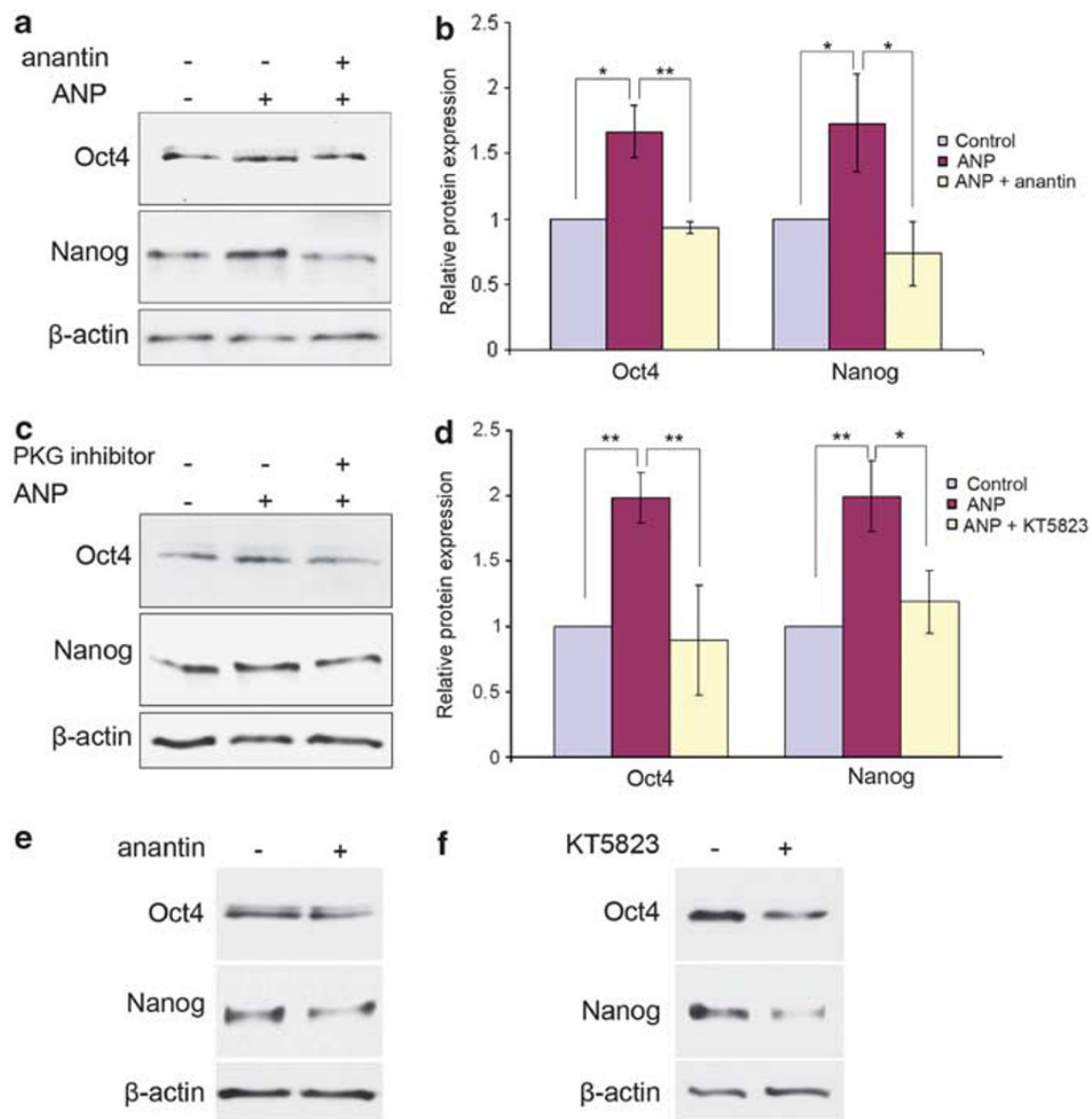

Figure 6 ANP upregulates Oct4 and Nanog through the NPR-A/PKG-dependent pathway. (a) Representative protein blot of Oct4 and Nanog proteins in ES cells treated with vehicle, ANP alone or ANP and anantin. (b) Quantitative analysis of the western blots as shown in panel a. (c) Representative protein blot of Oct4 and Nanog proteins in ES cells treated with vehicle, ANP alone or ANP and a PKG inhibitor (KT5823). (d) Quantitative analysis of the western blots as shown in panel c. (e) Western blot analysis of Oct4 and Nanog in ES cells treated with anantin for 3 days. (f) Western blot analysis of Oct4 and Nanog in ES cells treated with KT5823 for 3 days. Data represent mean \pm S.D. $(n=3) .{ }^{*} P<0.05$ or ${ }^{\star *} P<0.01$ (two-tailed $t$-test)

Therefore, we investigated the role of NPR-A in ES cells by examining the effect of NPR-A knockdown, which markedly induced differentiation of ES cells in the presence of LIF. Conversely, exogenous ANP enhanced ES cell pluripotency by inducing the expression of Oct4 and Nanog mRNA and protein in serum-free media. The positive effect of ANP on the expression of pluripotency markers was mainly dependent on ANP binding to its specific receptor, NPR-A, involving the cGMP/PKG pathway, as pretreatment with a specific NPR-A antagonist (anantin) or a PKG inhibitor abrogated ANPinduced upregulation of Oct4 and Nanog. These two transcription factors, Oct4 and Nanog, work in concert to maintain the undifferentiated state of ES cells. ${ }^{4,5,7}$

As key regulators of ES cell self-renewal and pluripotency, reducing either Nanog or Oct4 levels in ES cells triggers differentiation. ${ }^{9}$ Oct4 is a crucial regulator of ES cell pluripotency, and acts as a gatekeeper to prevent ES cell differentiation. ${ }^{27}$ It has been shown that reduction of Oct4 expression below $50 \%$ of normal levels induces differentiation of ES cells into the trophectoderm by upregulating the transcription factor $\mathrm{Cdx2} .{ }^{4,28} \mathrm{Cdx} 2$ and Oct4 also antagonize each other at the transcription level. ${ }^{29}$ In this study, knockdown of NPR-A markedly reduced Oct4 expression and induced the expression of the trophectodermal markers, $\mathrm{Cdx} 2$, Hand1 and Eomes. These findings suggest that the ANP/NPR-A system has a role in preventing the differentiation of ES cells into the trophectoderm by regulating Oct4 expression.

In murine ES cells, Nanog has been shown to block the differentiation of ES cells into extraembryonic endoderm cells by unknown mechanisms. ${ }^{5,7}$ In addition, knockdown of Nanog upregulates the expression of trophectodermal markers. ${ }^{28}$ Overexpression of Nanog in mouse ES cells can maintain them in a pluripotent state in the absence of LIF. ${ }^{5,7}$ In this study, knockdown of NPR-A markedly reduced the Nanog expression at mRNA and protein levels, and induced the expression of extraembryonic endoderm and trophectodermal markers. In addition, treatment of ES cells with ANP induced Nanog expression through an NPR-A-dependent pathway, and ANP was able to maintain the Nanog expression in the presence of the PI3K inhibitor. Thus, we speculate that ANP/NPR-A in ES cells contributes to maintaining the undifferentiated state and supports ES cell self-renewal by regulating Nanog expression.

Several of the known signaling pathways required for selfrenewal are also known to regulate the cell cycle, suggesting a 
link between control of cell proliferation and self-renewal. ${ }^{30}$ We found that knockdown of NPR-A inhibited ES cell proliferation and induced cell-cycle arrest at the G1 phase. Cell-cycle progression is controlled by cyclin-dependent kinases, ${ }^{31}$ and it is known that p21 has a critical role in the regulation of the G1-S transition, in which it works as an inhibitor of the G1 cyclin-dependent kinase-cyclin complexes. ${ }^{31}$ Consistent with these findings, NPR-A knockdown led to an upregulation in the p21 transcript. Recently, it has been found that Oct4 downregulation in ES cells upregulates p21 and blocks cell-cycle progression in G0/G1, leading to growth arrest, ${ }^{32}$ suggesting that suppression of the G1-S transition in NPR-A knockdown ES cells may be a consequence of the downregulation of Oct4 expression.

ES cells are known to express D-type cyclins at very low levels; expression of cyclin D1 is regulated by the PI3Kdependent pathway, with a critical role in the G1-S transition and maintenance of ES cell pluripotency. ${ }^{25,26,33}$ Our data also demonstrated that cyclin D1 and phosphorylated Akt are downregulated as a result of NPR-A knockdown. In addition, ES cells treated with ANP showed an increase in phosphorylated Akt, which is a downstream target of PI3K. Inhibition of $\mathrm{PI}$ KK/Akt signaling in mouse ES cells suppresses proliferation and induces differentiation of ES cells in the presence of LIF. ${ }^{25,26,33}$ Furthermore, the PI3K signaling pathway has been linked to ANP- and NPR-A-mediated cell proliferation in endothelial cells. ${ }^{34}$ These findings suggest that the PI3K pathway is involved in the effect of the ANP/NPR-A signaling system on ES cell pluripotency and self-renewal.

Although ANP and BNP share a common receptor (NPR-A), ${ }^{35}$ we found that ANP and BNP have different functions in ES cells. In this study, ANP activated ES cell pluripotency factors (namely Oct4 and Nanog) by its binding to NPR-A. Furthermore, NPR-A knockdown led to differentiation of ES cells. However, we previously demonstrated a role for BNP signaling in the maintenance of murine ES cell proliferation without affecting ES cell pluripotency. ${ }^{24}$ These results suggest that the effect of BNP in ES cells may be due to its binding to NPR-B and not to NPR-A. There are several studies showing similar findings in different cell types. For example, it has been reported that mice with targeted deletion of $\mathrm{BNP}^{36}$ exhibit a different phenotype than do ANP-deficient mice. ${ }^{37}$ This has led to the speculation that there may be a separate unknown receptor for BNP in cardiac fibroblasts. ${ }^{38}$ In addition, BNP increases bone growth by signaling through NPR-B,${ }^{19,38}$ which is not affected by ANP. ${ }^{39}$ Our findings from a combination of ANP addition and NPR-A inhibition experiments provide substantial evidence to support the hypothesis that ANP signaling through NPR-A has a role in repressing ES cell differentiation.

In conclusion, we have demonstrated here for the first time that expression and activation of NPR-A are essential for maintaining self-renewal and pluripotency of ES cells. Knockdown of NPR-A represses the expression of genes encoding the pluripotency factors, which would subsequently lead to differentiation of ES cells. Our study also shows that exogenous ANP positively regulates ES cell pluripotency, involving its receptor NPR-A in a PKG-dependent manner (Figure 7). Thus, maintaining an appropriate level of endogenous NPR-A is critical for ES cell pluripotency.

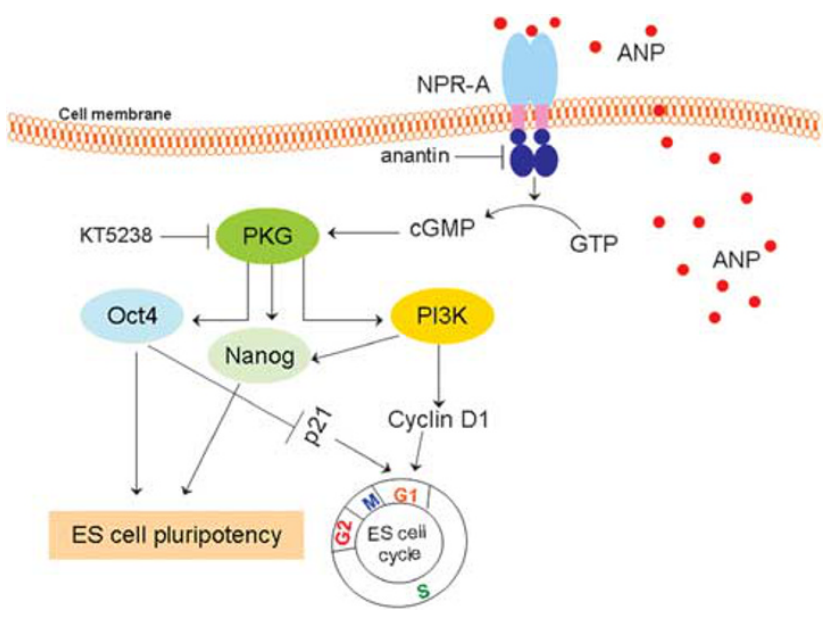

Figure 7 Proposed model of NPR-A signaling-mediated maintenance of ES cell self-renewal and pluripotency. Activation of NPR-A by ANP stimulates the CGMP/ PKG signaling pathway, leading to increased expressions of Oct4 and Nanog, and reduced expression of p21, which in turn suppresses ES cell differentiation and maintains ES cell pluripotency. In another pathway, binding of ANP to NPR-A activates the phosphoinositide 3-kinase (PI3K) signaling pathway, which enhances G1-to-S progression through induction of cyclin D1, and maintains pluripotency by activation of Nanog expression

The findings reported here provide novel molecular mechanisms involved in the self-renewal and pluripotency of ES cells.

\section{Materials and Methods}

ES cell culture. Murine ES cells (E14TG2a) (CRL-1821; American Type Culture Collection, Manassas, VA, USA) were maintained in DMEM/F-12 medium (Sigma, St. Louis, MO, USA), which was supplemented with $1000 \mathrm{Units} / \mathrm{ml}$ LIF (Chemicon, Temecula, CA, USA), $11 \%$ fetal bovine serum, $2 \mathrm{mM}$ L-glutamine (Nacalai Tesque, Kyoto, Japan), $1 \mathrm{mM}$ sodium pyruvate (Sigma), 1\% MEM nonessential amino acids (Gibco, Carlsbad, CA, USA), $0.1 \mathrm{mM}$ 2-mercaptoethanol (Sigma) and 1\% penicillin-streptomycin. For siRNA transfection, ES cells were cultured in the same medium, except that fetal bovine serum was replaced with $15 \%$ knockout serum replacement (Gibco). Murine ES cells were cultured under feederfree conditions in the presence of LIF. For differentiation, EBs were grown in bacteriologic dishes without LIF for 4-5 days. Rat ANP (Sigma), anantin (Bachem, Torrance, CA, USA), KT5823 (Calbiochem) and LY294002 (Calbiochem) were added to cultured ES cells at 2.5, 300, 2 and $10 \mu \mathrm{M}$ respectively, whereas control cells received vehicle alone.

In vitro differentiation. Undifferentiated ES cells were trypsinized and resuspended in the ES cell culture medium without LIF. Differentiation was induced by the hanging drop method as a standard method of EB formation, and treatment with RA for 3 days. EBs were further cultured for 7 days in a differentiation medium.

Immunofluorescence. ES cells grown on glass coverslips or 35-mm dishes were rinsed briefly with phosphate-buffered saline (PBS) and fixed for $20 \mathrm{~min}$ in $4 \%$ paraformaldehyde in $0.1 \mathrm{M}$ phosphate buffer ( $\mathrm{pH}$ 7.4). ES cells were permeabilized for 10 min with $0.1 \%$ Triton X-100 in PBS, and blocked for 40 min with $4 \%$ bovine serum albumin in PBS at room temperature. They were then incubated overnight at $4^{\circ} \mathrm{C}$ with the following antibodies: anti-ANP (1:500; Peptide Osaka Institute, Osaka, Japan), anti-ANP (1:300; sc-18811; Santa Cruz Biotechnology, Santa Cruz, CA, USA), anti-NPR-A (1:300; Abcam, Cambridge, UK), anti-Oct4 (1:200, sc-5279; Santa Cruz Biotechnology) and anti-Nanog (1:2000, Bethyl Laboratories, Montgomery, TX, USA). This was followed by incubation with the following secondary antibodies: Alexa Fluor 594-labeled anti-goat IgG, Alexa Fluor 594labeled anti-mouse IgG, Alexa Fluor 488-labeled anti-mouse IgG and Alexa Fluor 488-labeled anti-rabbit lgG (all $1: 400$; Molecular Probes, Paisley, UK). Nuclei were counterstained using Hoechst $33342(1 \mu \mathrm{g} / \mathrm{ml}$; Invitrogen, Carlsbad, CA, USA). 
The slides were examined by fluorescence microscopy (Biozero; Keyence, Osaka, Japan) or by confocal laser microscopy (C1si; Nikon, Tokyo, Japan), and images were processed using Nikon EZ-C1 viewer software.

RT-PCR and real-time PCR. Total RNA was extracted using the RNeasy Mini kit (Qiagen, Hilden, Germany) according to the manufacturer's instructions. cDNA synthesis was performed with $1 \mu \mathrm{g}$ of total RNA using the Superscript III firststrand cDNA synthesis kit (Invitrogen). The cDNA $(0.5 \mu \mathrm{g})$ was used as a template in a mixture that contained Ampli-Taq Gold polymerase (Applied Biosystems, Foster City, CA, USA). RT-PCR was performed with an annealing temperature of $58^{\circ} \mathrm{C}$. cDNA samples were analyzed by real-time PCR in a LightCycler Real-Time PCR System (Roche Diagnostics, Tokyo, Japan) using the SYBR Premix Ex Taq II (Takara, Otsu, Japan)

The level of the different mRNAs was normalized to that of the housekeeping gene, $\beta$-actin (see Supplementary Table 1 for primer sequences). A comparative $\mathrm{Ct}$ (cycle time) analysis was used to determine fold changes in mRNA expression based on the $\Delta \Delta \mathrm{Ct}$ approach. ${ }^{40}$ The reactions were carried out in triplicate.

RNA interference. RNA interference in murine ES cells was carried out according to the manufacturer's protocol using Lipofectamine RNAiMAX (Invitrogen) in 6-well plates. Two pairs of siRNAs (Invitrogen) were designed for NPR-A (NM_008727) using the BLOCK-iT RNAi Designer software (Invitrogen). The appropriate siRNA-negative control Duplex (category no. 12935-300; Invitrogen) was selected based on the percentage G/C. NPR-A siRNA and control siRNA were transfected at a final concentration of $40 \mu \mathrm{M}$ for $24 \mathrm{~h}$ in triplicate for each treatment. At $48 \mathrm{~h}$ after transfection, NPR-A knockdown was confirmed by RT-PCR, real-time PCR and western blotting. The sequences of the NPR-A siRNAs were as follows:

NPR-A siRNA1: sense, 5'-AGAUGUAGAUAACUCUGCCCUUGCG-3', antisense, 5'-CGCAAGGGCAGAGUUAUCUAUACAUCU-3'; NPR-A siRNA2: sense, 5'-GCCUUCAGGGUUGUCCUGAACUUUA-3'; antisense, 5'-UAAAGUUCAGGA CAACCCUGAAGGC-3'.

Western blotting. Total protein extracts were prepared from ES cells, dissolved in SDS-PAGE buffer and transferred to nitrocellulose membranes (Amersham Biosciences, Freiburg, Germany). Proteins were detected using antibodies against NPR-A (1:1000, sc-31632; Santa Cruz Biotechnology), phospho-Akt (1:1000, Ser473; Cell Signaling, Beverly, MA, USA), Akt (1:1000 Ser473; Cell Signaling), Oct4 (1 : 1000, sc-5279; Santa Cruz Biotechnology), Nanog (1: 8000, A300-397A; Bethyl Laboratories), ANP (1:1000, sc-18811; Santa Cruz Biotechnology), p21 (1:1000, sc-397; Santa Cruz Biotechnology) and $\beta$-actin (1:8000, sc-47778; Santa Cruz Biotechnology). The secondary antibodies were peroxidase-conjugated anti-rabbit IgG, peroxidase-conjugated anti-goat IgG or peroxidase-conjugated anti-mouse IgG (all 1:10000; Jackson ImmunoResearch Lab Inc., Newmarket, UK). Blots were developed using SuperSignal West Pico Chemiluminescent substrate (Pierce, Loughborough, UK), and visualized using an LAS-3000 Fujifilm Lumino-Image Analyzer (FujiFilm, Tokyo, Japan).

Self-renewal assays. To determine the ability of ES cells to retain an undifferentiated phenotype, self-renewal assays were performed using an AP kit (Chemicon) as described previously. ${ }^{24}$ AP-positive colonies were counted by microscopic examination. Colonies of tightly packed and flattened AP-positive cells were considered undifferentiated, and colonies of mixtures of unstained and stained cells and entirely unstained cells with flattened irregular morphology were considered differentiated.

Cell-cycle analysis. Cells were fixed overnight in $70 \%$ ethanol at $4^{\circ} \mathrm{C}$. Enzymatic removal of RNA was carried out using $100 \mu \mathrm{g} / \mathrm{ml}$ RNase (Boehringer Mannheim GmbH, Mannheim, Germany) at room temperature for $20 \mathrm{~min}$. Cells were then stained with $5 \mu \mathrm{g} / \mathrm{ml}$ propidium iodide (Sigma) at $4^{\circ} \mathrm{C}$ for $40 \mathrm{~min}$. Flowcytometric analysis was carried out on 20000 gated events. Data acquisition was performed using a FACSCalibur (BD Biosciences, Oxford, UK), and cell-cycle phase distribution was analyzed using ModFit LT flow cytometry modeling software (Verity Software House, Topsham, ME, USA)

Cell proliferation assay. To determine the number of cells, cells were washed with PBS and trypsinized from the culture dishes. Cell suspension was mixed with trypan blue solution, and the number of live cells was determined using a homocytometer. Cells failing to exclude the dye were considered nonviable.
Statistical analysis. The results are expressed as mean \pm S.D., as indicated in the figure legends. Statistical significance was assessed by two-tailed Student's $t$-tests. Values of $P<0.05$ were considered significant.

\section{Conflict of interest}

The authors declare no conflict of interest.

Acknowledgements. This work was supported by a Grant-in-Aid for Scientific Research (No. 21-09133) from the Japan Society for the Promotion of Science.

1. Evans MJ, Kaufman MH. Establishment in culture of pluripotent potential cells from mouse embryos. Nature 1981; 292: 154-156.

2. Niwa H. How is pluripotency determined and maintained? Development 2007; 134 635-646.

3. Burdon T, Smith A, Savatier P. Signalling, cell cycle and pluripotency in embryonic stem cells. Trends Cell Biol 2002; 12: 432-438.

4. Niwa H, Miyazaki J, Smith AG. Quantitative expression of Oct-3/4 defines differentiation, dedifferentiation or self-renewal of ES cells. Nature Genet 2000; 24: 372-376.

5. Mitsui K, Tokuzawa $\mathrm{Y}$, Itoh $\mathrm{H}$, Segawa K, Murakami M, Takahashi $\mathrm{K}$ et al. The homeoprotein Nanog is required for maintenance of pluripotency in mouse epiblast and ES cells. Cell 2003; 113: 631-642.

6. Avilion AA, Nicolis SK, Pevny LH, Perez L, Vivian N, Lovell-Badge R. Multipotent cell lineages in early mouse development depend on SOX2 function. Genes Dev 2003; 17 : $126-140$.

7. Chambers I, Colby D, Robertson M, Nichols J, Lee S, Tweedie S et al. Functional expression cloning of Nanog a pluipotency sustaining factor in embryonic stem cells. Cell 2003; 113: 643-655.

8. Loh YH, Wu Q, Chew JL, Vega VB, Zhang W, Chen X et al. The Oct4 and Nanog transcription network regulates pluripotency in mouse embryonic stem cells. Nat Genet 2006; 38: 431-440.

9. Chambers I, Smith A. Self-renewal of teratocarcinoma and embryonic stem cells. Oncogene 2004; 23: 7150-7160.

10. Levin ER, Gardner DG, Samson WK. Natriuretic peptides. N Engl J Med 1998; 339 : 321-328.

11. Pandey KN. Biology of natriuretic peptides and their receptors. Peptides 2005; 26: 901-932.

12. Morishita R, Gibbons GH, Pratt RE, Tomita N, Kaneda Y, Ogihara T et al. Autocrine and paracrine effects of atrial natriuretic peptide gene transfer on vascular smooth muscle and endothelial cellular growth. J Clin Invest 1994; 94: 824-829.

13. Wu CF, Bishopric NH, Pratt RE. Atrial natriuretic peptide induces apoptosis in neonatal rat cardiac myocytes. J Biol Chem 1997; 272: 14860-14866.

14. Kato T, Muraski J, Chen Y, Tsujita Y, Wall J, Glembotski CC et al. Atrial natriuretic peptide promotes cardiomyocyte survival by cGMP-dependent nuclear accumulation of zyxin and Akt. J Clin Invest 2005; 115: 2716-2730.

15. Cao L, Gardner DG. Natriuretic peptides inhibit DNA synthesis in cardiac fibroblasts. Hypertension 1995; 25: 227-234.

16. You $\mathrm{H}$, Laychock SG. Atrial natriuretic peptide promotes pancreatic islet $\beta$-cell growth and Akt/Foxo1a/cyclin D2 signaling. Endocrinology 2009; 150: 5455-5465.

17. Kong X, Wang X, Xu W, Behera S, Hellermann G, Kumar A et al. Natriuretic peptide receptor a as a novel anticancer target. Cancer Res 2008; 68: 249-256.

18. Silberbach M, Roberts Jr CT. Natriuretic peptide signaling: molecular and cellular pathways to growth regulation. Cell Signal 2001; 13: 221-231.

19. Yasoda A, Ogawa Y, Suda M, Tamura N, Mori K, Sakuma Y et al. Natriuretic peptide regulation of endochondral ossification. Evidence for possible roles of the $\mathrm{C}$-type natriuretic peptide/guanylyl cyclase-B pathway. J Biol Chem 1998; 273: 11695-11700.

20. Oliver PM, Fox JE, Kim R, Rockman HA, Kim HS, Reddick RL et al. Hypertension, cardiac hypertrophy, and sudden death in mice lacking natriuretic peptide receptor A. Proc Nalt Acad Sci USA 1997; 94: 14730-14735.

21. Kuhn M, Holtwick R, Baba HA, Perriard JC, Schmitz W, Ehler E. Progressive cardiac hypertrophy and dysfunction in atrial natriuretic peptide receptor (GC-A) deficient mice. Heart 2002; 87: 368-374.

22. Ellermers LJ, Knowles JW, Kim HS, Smithies O, Maeda N, Cameron VA. Ventricular expression of natriuretic peptide in $\mathrm{Npr}(-)$ mice with cardiac hypertrophy and fibrosis. Am J Physiol Heart Circ Physiol 2002; 283: H707-H714.

23. Scott NJA, Ellmers LJ, Lainchbury JG, Maeda N, Smithies O, Richards AM et al. Influence of natriuretic peptide receptor-1 on survival and cardiac hypertrophy during development. Biochimica et Biophysica Acta 2009; 1792: 1175-1184

24. Abdelalim EM, Tooyama I. BNP signaling is crucial for embryonic stem cell proliferation. PloS One 2009; 4: e35341.

25. Paling NR, Wheadon H, Bone HK, Welham MJ. Regulation of embryonic stem cell selfrenewal by phosphoinositide 3-kinase-dependent signalling. J Biol Chem 2004; 279: 48063-48070. 
26. Storm MP, Bone HK, Beck CG, Bourillot PY, Schreiber V, Damiano T et al. Regulation of Nanog expression by phosphoinsitide 3-kinase-dependent signaling in murine embryonic stem cells. J Biol Chem 2007; 282: 6265-6273.

27. Nichols J, Zevnik B, Anastassiadis K, Niwa H, Klewe-Nebenius D, Chambers I et al. Formation of pluripotent stem cells in the mammalian embryo depends on the POU transcription factor Oct4. Cell 1998; 95: 379-391.

28. Chen L, Yabuuchi A, Eminli S, Takeuchi A, Lu CW, Hochedlinger K et al. Cross-regulation of the Nanog and Cdx2 promoters. Cell Res 2009; 19: 1052-1061.

29. Niwa H, Toyooka $Y$, Shimosato D, Strumpf D, Takahashi K, Yagi R et al. Interaction between Oct3/4 and Cdx2 determines trophectoderm differentiation. Cell 2005; 123: 917-929.

30. White J, Dalton S. Cell cycle control of embryonic stem cells. Stem Cell Rev 2005; 1: 131-138.

31. Harper JW, Adami GR, Wei H, Keyomarsi K, Elledge SJ. The p21 Cdk-interacting protein Clip1 is a potent inhibitor of G1 cyclin-dependent kinases. Cell 1993; 75: 805-816.

32. Lee J, Go Y, Kang I, Han YM, Kim J. Oct-4 controls cell cycle progression of embryonic stem cells. Biochem J 2010; 426: 171-181.

33. Jirmanova L, Afanassieff M, Gobert-Gosse S, Markossian S, Savatier P. Differential contributions of ERK and PI3-kinase to the regulation of cyclin D1 expression and to the control of the G1/S transition in mouse embryonic stem cells. Oncogene 2002; 21: $5515-5528$.

34. Kook H, Itoh H, Choi BS, Sawada N, Doi K, Hwang TJ et al. Phsyiological concentration of atrial natriuretic peptide induces endothelial regeneration in vitro. Am J Physiol Heart Circ Physiol 2003; 284: H1388-H1397.
35. Suga S, Nakao K, Hosoda K, Mukoyama M, Ogawa Y, Shirakami G et al. Receptor selectivity of natriuretic peptide family, atrial natriuretic peptide, brain natriuretic peptide, and C-type natriuretic peptide. Endocrinology 1992; 130: 229-239.

36. Tamura N, Ogawa Y, Chusho H, Nakamura K, Nakao K, Suda M et al. Cardiac fibrosis in mice lacking brain natriuretic peptide. Proc Nalt Acad Sci USA 2000; 97: 4239-4244

37. John SW, Krege JH, Oliver PM, Hagaman JR, Hodgin JB, Pang SC et al. Genetic decreases in atrial natriuretic peptide and salt-sensitive hypertension. Science 1995; 267 679-681.

38. Suda M, Ogawa Y, Tanaka K, Tamura N, Yasoda A, Takigawa T et al. Skeletal overgrowth in transgenic mice that overexpress brain natriuretic peptide. Proc Natl Acad Sci USA 1998; 95: 2337-2342.

39. Steinhelper ME, Cochrane KL, Field LJ. Hypotension in transgenic mice expressing atrial natriuretic factor fusion genes. Hypertension 1990; 16: 301-307.

40. Livak KJ, Schmittgen TD. Analysis of relative gene expression data using real-time quantitative PCR and the $2^{-\Delta \Delta C T}$ method. Methods 2001; 25: 402-408.

Cell Death and Disease is an open-access journal published by Nature Publishing Group. This work is licensed under the Creative Commons Attribution-Noncommercial-No Derivative Works 3.0 Unported License. To view a copy of this license, visit http://creativecommons.org/licenses/by-nc-nd/3.0/

Supplementary Information accompanies the paper on Cell Death and Disease website (http://www.nature.com/cddis) 